\title{
Doença de Mucha-Habermann (variante febril úlcero-necrótica) com acometimento mucoso exuberante - Relato de caso
}

\author{
Febrile ulceronecrotic Mucha-Habermann disease with exuberant mucosal \\ involvement - Case report
}

\author{
Moema Mignac Cumming \\ Mário Alberto S. Paino ${ }^{3}$ \\ Ana Maria Roselino5
}

\author{
Adriana S. Salathiel ${ }^{2}$ \\ Sérgio Delort ${ }^{4}$
}

\begin{abstract}
Resumo: A pitiríase liquenoide é dermatose incomum, idiopática, com espectro clínico-histopatológico onde inclui a forma varioliforme aguda (doença de Mucha-Habermann), sua variante febril úlceronecrótica e a forma crônica. Manifestações sistêmicas podem ocorrer na variante febril úlcero-necrótica, com relatos de casos fatais em adultos.

Relata-se o caso de um paciente jovem, com diagnóstico clínico e histopatológico de doença de MuchaHabermann, variante febril úlcero-necrótica, e acometimento mucoso exuberante, fato ocasional, mesmo nas formas mais graves de pitiríase liquenoide. Além do aspecto clínico inusitado, demonstra-se excelente resultado terapêutico, com a associação de prednisona e metotrexato.

Palavras-chave: Metotrexato; Pitiríase liquenóide; Prednisona

Abstract: Pityriasis lichenoides is a rare idiopathic cutaneous disorder, with a clinical-histopathological spectrum comprising the acute varioliform form (Mucha-Habermann's disease), its febrile ulceronecrotic variant, and its chronic form. Systemic manifestations may occur in the febrile ulceronecrotic variant, with reports of adult mortality.

The case of a young male patient with clinical and histopathological diagnosis of Mucha-Habermann's disease, febrile ulceronecrotic variant, with severe mucosal involvement - an occasional incidence even in the most severe forms of pityriasis lichenoides - is presented. In addition to the atypical clinical aspect, an excellent therapeutic result is shown with the association of prednisone and methotrexate.

Keywords: Methotrexate; Pityriasis lichenoides; Prednisone
\end{abstract}

\section{INTRODUÇÃO}

A pitiríase liquenoide varioliforme aguda (PLEVA), também conhecida como doença de Mucha-Habermann, é dermatose incomum, idiopática, caracterizada por lesões eritematosas, descamativas, frequentemente, acompanhadas de lesões pápulo-necróticas e hemorrágicas, pois tipicamente evoluem para cicatrizes atróficas e discrômicas. ${ }^{1}$ Afetam tanto crianças quanto adultos, nas primeiras décadas de vida. A rara variante febril úlcero-necrótica foi, inicialmente, descrita por Degos, em 1966, e caracterizase por início repentino de pápulas purpúricas disseminadas, com úlceras de centro necrótico e manifestações sistêmicas. ${ }^{1-3}$

Recebido em 20.8.2008.

Aprovado pelo Conselho Consultivo e aceito para publicação em 6.11.09.

* Trabalho realizado no Serviço de Dermatologia do Hospital das Clínicas da Faculdade de Medicina de Ribeirão Preto - (FMRP/ USP) - Ribeirão Preto (SP), Brasil.

Trabalho premiado como pôster de Caso Clínico no Congresso de Dermatologia de 2008.

Conflito de interesse: Nenhum / Conflict of interest: None

Suporte financeiro: Nenhum / Financial funding: None

Especialista em Dermatologia - Ribeirão Preto (SP), Brasil

Pós graduanda - nível Doutorado - FMRP-USP - Ribeirão Preto (SP), Brasil.

Médico Patologista do Laboratório de Anatomia Patológica Dr. Mário A. S. Paino - Araraquara (SP), Brasil.

Médico Dermatologista, Presidente do VI Distrito Dermatológico de Ribeirão Preto - Ribeirão Preto (SP), Brasil.

Professora Associada da Divisão de Dermatologia, Departamento de Clínica Médica, Faculdade de Medicina de Ribeirão Preto, Universidade de São Paulo São Paulo (SP), Brasil.

CC2009 by Anais Brasileiros de Dermatologia 


\section{RELATO DO CASO}

Paciente de 21 anos, sexo masculino, natural e procedente do estado de São Paulo, universitário, solteiro, referia, nos últimos seis meses, episódios recorrentes de infecção de vias aéreas superiores, tendo feito uso prévio de antibióticos, com resolução do quadro. Procurou serviço médico em novembro de 2007, com relato de que há 45 dias apresentava lesão ulcerada, no lábio inferior, seguida por surgimento de lesões eritematosas não-pruriginosas no tronco, membros e cavidade oral, acompanhadas por picos febris $\left(38,5^{\circ} \mathrm{C}\right)$, mal-estar geral e artralgia.

Ao exame dermatológico, notavam-se pápulas eritematosas no tronco, membros, palmas e plantas, muitas delas centradas por descamação em retalho, sem vesículas ou bolhas íntegras. Notavam-se também lesões úlcero-necróticas, algumas de aspecto varioliforme, lenticulares a numulares, no dorso, glúteos, membros e genitália (Figura 1).

O paciente foi tratado com antibioticoterapia e prednisona $0,3 \mathrm{mg} / \mathrm{kg} / \mathrm{dia}$, além de cuidados tópicos, em outro serviço. Com piora do quadro cutâneo, foi aumentada a dose de prednisona para $1 \mathrm{mg} / \mathrm{kg} / \mathrm{dia}$ e ampliado espectro antimicrobiano. Como não houvesse resposta clínica, foi encaminhado a este serviço, onde exames laboratoriais gerais foram normais; sorologias para hepatites B e C, HIV e vírus Epstein-Barr não eram reagentes, assim como para outras infecções virais. Culturas para agentes microbianos resulta- ram negativas e exames laboratoriais para vasculites (incluindo ANCA) excluíram esta hipótese. Estudo anatomopatológico evidenciou epiderme com exocitose de linfócitos sem atipias, necrose de ceratinócitos e dermatite de interface vacuolar; derme com moderado infiltrado linfocitário, superficial e profundo, perivascular e intersticial, com extravasamento de hemácias (Figura 2). Com a hipótese de PLEVA, forma febril úlcero-necrótica, manteve-se a dose de prednisona, sendo associado metotrexato, na dose semanal de $15 \mathrm{mg}$ via oral, com melhora significativa em seis semanas. Após quatro meses de tratamento, evoluiu com resolução do quadro clínico, sendo suspensa a terapêutica instituída. Apresentou novo surto, mais brando, há uma semana, quando foi reintroduzido o mesmo tratamento. Ainda apresenta cicatrizes atróficas, de aspecto varioliforme (Figura 3).

\section{DISCUSSÃO}

A doença de Mucha-Habermann, forma febril úlcero-necrótica, é a variante mais destrutiva da pitiríase liquenoide, com presença de ulcerações coalescentes e febre alta. ${ }^{2-4}$ Sintomas associados incluem: artralgia, acometimento do trato gastrintestinal e do sistema nervoso central, pneumonite intersticial e miocardite linfocítica, podendo levar ao óbito. A distribuição das lesões é similar à PLEVA, com envolvimento ocasional da mucosa oral. ${ }^{1,3}$ Sua etiologia per-
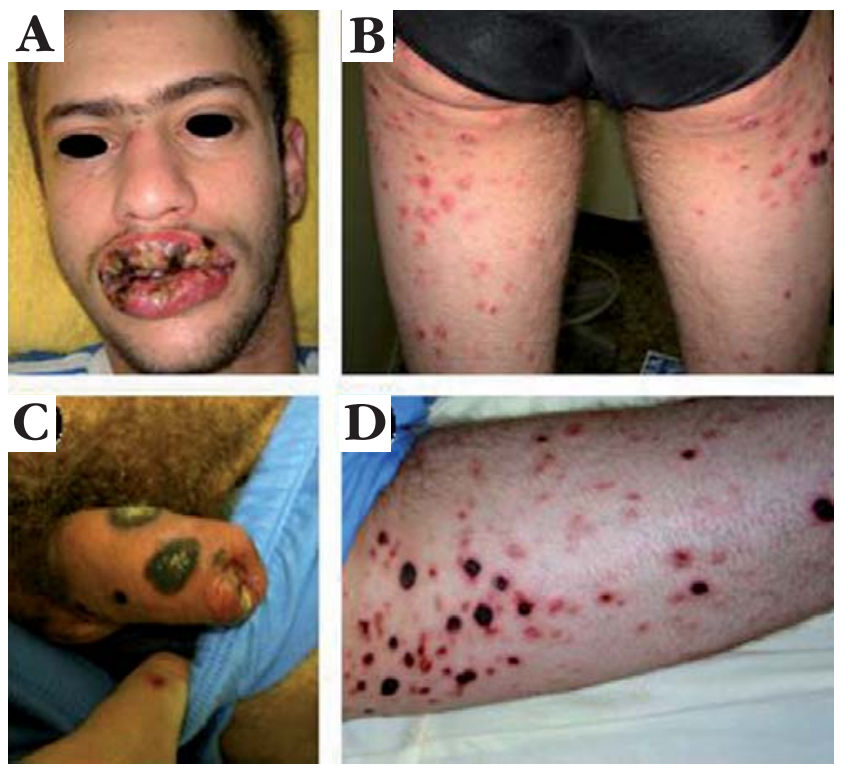

Figura 1: Lesões dermatológicas em paciente com PLEVA. A. Ulcerações com áreas necróticas coalescentes nos lábios superior e inferior, associadas ao pronunciado edema; B. Pápulas eritematosas, lesões descamativas e úlcero-necróticas, na face posterior das coxas; C. Edema de glande e prepúcio, com numerosas lesões úlcero-necróticas na bolsa escrotal e corpo peniano; D. Lesões pápulo-necróticas na face anterior de coxa direita
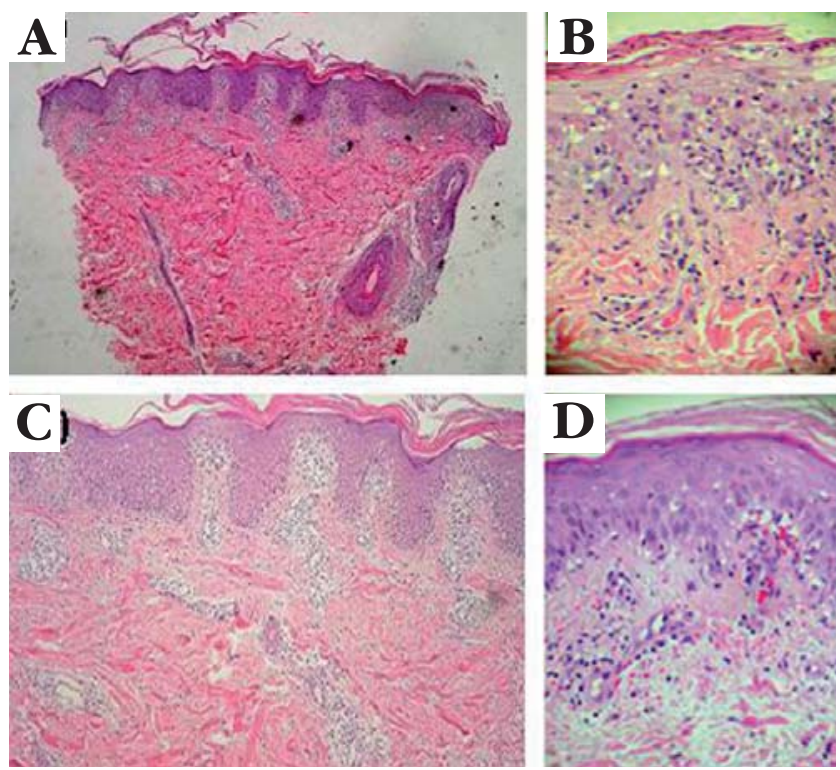

Figura 2: Biópsia de pele: Dermatite de interface vacuolar com exocitose de linfócitos e ceratinócitos necróticos. Proliferação vascular na derme papilar e média, com infiltrado linfocitário perivascular superficial e profundo, além de extravasamento de hemácias (H\&E; A. 40x; B. 100x; C. e D. 200x) 


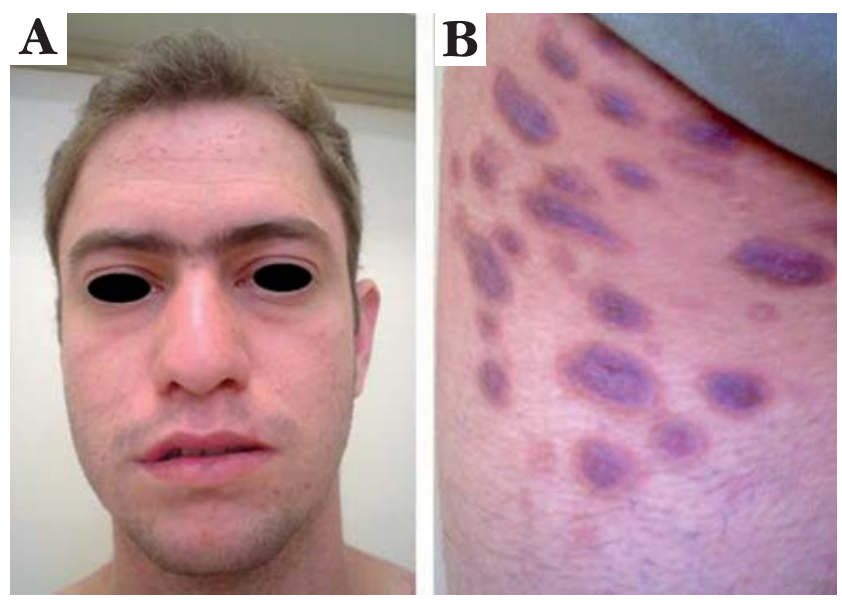

Figura 3: Evolução do quadro dermatológico, em paciente com PLEVA: A. e B. Oitava semana, após introdução do metotrexato e manutenção de prednisona, apresentando resolução das lesões cutâneas, com lesões residuais francamente varioliformes

manece desconhecida, porém a hipótese mais amplamente aceita atualmente indica as reações de hipersensibilidade a agentes infecciosos como mecanismos de base para o desenvolvimento da PLEVA. Na literatura, vários patógenos têm sido associados à doença, incluindo HIV, vírus da varicela-zoster, vírus EpsteinBarr, citomegalovírus, adenovírus, Staphylococcus,
Streptococcus, Mycoplasma e Toxoplasma gondii. ${ }^{1,2,5}$ Para o caso ora relatado, havia antecedentes de infecções repetidas do trato respiratório superior precedendo, em seis meses, a primeira lesão necrótica labial. A pesquisa de bactérias e as sorologias virais resultaram negativas, não sendo possível relacioná-las à PLEVA.

Alguns relatos têm demonstrado a presença de populações clonais de linfócitos $\mathrm{T}$ no sangue e em lesões cutâneas de pacientes com PLEVA, sugerindo que a doença seja desordem linfoproliferativa de células T, a despeito da ausência de linfócitos morfologicamente atípicos nas lesões cutâneas. ${ }^{3,5,6}$ Essa seria outra teoria usada por muitos autores, para tentar explicar a etiologia da doença.

Para a variante úlcero-necrótica, existem relatos de resultados favoráveis com o uso de glicocorticoides sistêmicos, combinação de fototerapia e metotrexato oral, metotrexato intravenoso, ciclosporina e gamaglobulina. ${ }^{7-10}$ Levando-se em consideração o seu curso potencialmente grave, faz-se necessária monitoração rigorosa do paciente, para prevenção de desfechos fatais.

Nesse relato de caso, chama atenção o acometimento mucoso exuberante, fato incomum, mesmo nas formas mais graves de PLEVA, aliado à resposta favorável ao corticoide com metotrexato.

\section{AGRADECIMENTOS}

À médica patologista, Dra. Catarina Shaletich, pela excelente revisão histopatológica, e à dentista Elaine Maria Sgavioli Massucato, Professora Doutora da Faculdade de Odontologia de Araraquara, UNESP, Departamento de Estomatologia, pelo encaminhamento do caso ao Dr. Delort (co-autor). 


\section{REFERÊNCIA}

1. Khachemoune A, Blyumin ML. Pityriasis lichenoides: pathophysiology, classification, and treatment. Am J Clin Dermatol. 2007;8:29-36.

2. Bowers S, Warshaw EM. Pityriasis lichenoides and its subtypes. J Am Acad Dermatol. 2006;55:557-72.

3. Miyamoto T, Takayama N, Kitada S, Hagari Y, Mihara M. Febrile ulceronecrotic MuchaHabermann disease: a case report and a review of the literature. J Clin Pathol. 2003;56:795-7.

4. Aytekin S, Balci G, Duzgun OY. Febrile ulceronecrotic Mucha-Habermann disease: a case report and a review of the literature. Dermatol Online J. 2005;11:31.

5. Hoshina D, Akiyama M, Hamasaka K, Shimizu H. An infantile case of pityriasis lichenoides et varioliformis acuta. Br J Dermatol. 2007;157:194-6.

6. Nair PS. A clinical and histopathological study of pityriasis lichenoides. Indian J Dermatol Venereol Leprol. 2007;73:100-2.

7. Malnar T, Milavec-Puretic V, Rados J, Zarcovic K, Dobric I. Febrile ulceronecrotic pityriasis lichenoids et et varioliformis acuta with fatal outcome. J Eur Acad Dermatol Venereol. 2006;20:303-7.
8. Pyrpasopoulou A, Athyros VG, Karagiannis A, Chrysomallis F, Zamboulis C. Intravenous immunoglobulins: a valuable asset in the treatment of a case of septic febrile ulceronecrotic Mucha-Habermann disease. Dermatology. 2007;215:164-5.

9. Kim HS, Yu DS, Kim JW. A case of febrile ulceronecrotic Mucha-Habermann's disease successfully treated with oral cyclosporin. J Eur Acad Dermatol Venereol. 2007;21:272-3.

10. Levy AL. Rapid resolution of pityriasis lichenoides et varioliformis acuta with azithromycin. J Am Acad Dermatol. 2008;58:524-5.

ENDEREÇO PARA CORRESPONDÊNCIA / MAILING ADDRESS: Ana Maria Roselino

Departamento de Clínica Médica - FMRP - USP.

Av. Bandeirantes 3900

14049900 - Ribeirão Preto - SP. Brasil

Tel.:/Fax: 551636336695

e-mail:amfrosel@fmrp.usp.br

Como citar este artigo / How to cite this article: Cumming MM, Salathiel ASM, Paino MAS, Delort S, Roselino AM. Doença de Mucha-Habermann (variante febril úlcero-necrótica) com acometimento mucoso exuberante - Relato de caso. An Bras Dermatol. 2009;84(6):655-8. 